\title{
A new surgical technique for congenital distichiasis
}

\section{Alicia Galindo-Ferreiro, Hind Alkatan, Azza Maktabi, Alberto Gálvez-Ruiz \& Silvana Schellini}

To cite this article: Alicia Galindo-Ferreiro, Hind Alkatan, Azza Maktabi, Alberto Gálvez-Ruiz \& Silvana Schellini (2017): A new surgical technique for congenital distichiasis, Orbit, DOI: 10.1080/01676830.2017.1383454

To link to this article: http://dx.doi.org/10.1080/01676830.2017.1383454

\section{Published online: 23 Oct 2017.}

Submit your article to this journal ¿

Q View related articles $\widetilde{x}$

View Crossmark data $\nearrow$ 


\title{
A new surgical technique for congenital distichiasis
}

\author{
Alicia Galindo-Ferreiro ${ }^{\mathrm{a}, \mathrm{b}}$, Hind Alkatan ${ }^{\mathrm{c}}$ Azza Maktabia, Alberto Gálvez-Ruiz ${ }^{\mathrm{a}}$, and Silvana Schellinia,d \\ aculoplastic Division, King Khaled Eye Specialist Hospital, Riyadh, Saudi Arabia; ${ }^{b}$ Department of Ophthalmology, Complejo Asistencial de \\ Palencia, Palencia, Spain; 'Department of Ophthalmology, King Saud University College of Medicine, Riyadh, Saudi Arabia; ${ }^{d}$ Department of \\ Ophthalmology Faculdade de Medicina de Botucatu, UNESP, São Paulo, Brasil
}

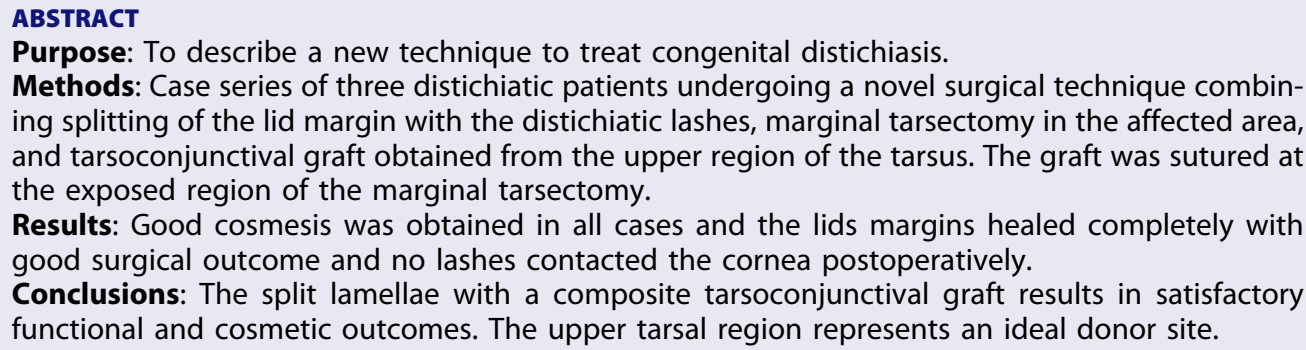
ing splitting of the lid margin with the distichiatic lashes, marginal tarsectomy in the affected area, and tarsoconjunctival graft obtained from the upper region of the tarsus. The graft was sutured at the exposed region of the marginal tarsectomy.

Results: Good cosmesis was obtained in all cases and the lids margins healed completely with good surgical outcome and no lashes contacted the cornea postoperatively.

Conclusions: The split lamellae with a composite tarsoconjunctival graft results in satisfactory functional and cosmetic outcomes. The upper tarsal region represents an ideal donor site.

\section{ARTICLE HISTORY}

Received 8 November 2016

Revised 3 July 2017

Accepted 19 Septemebr 2017

\section{KEYWORDS}

Congenital anomalies; eyelid disease; lashes; surgery

\section{Introduction}

Distichiasis is a congenital or acquired condition with autosomal dominant inheritance that was initially described in the 18th century. ${ }^{1-3}$ Distichiasis manifests as a partial or complete accessory row of lashes emerging from the ostia of the Meibomian glands, affecting one or both eyelids. ${ }^{1-3}$ The congenital form is more common and the acquired form is associated with chronic lid inflammation. ${ }^{4}$

Distichiasis can be isolated or occur in association with other conditions. ${ }^{1}$ Symptoms of distichiasis occur due to damage to the anterior surface of the globe caused by displaced lashes. ${ }^{1}$

There are numerous treatments for distichiasis including, epilation, electrolysis, lid margin cryotherapy, split lamella with or without cryotherapy in the posterior lamella, mucous membrane graft, or nasal condro-mucosal graft. ${ }^{1,2,5-11}$ However, none of these treatment are completely satisfactory. ${ }^{1,2,5-11}$

We describe a new surgical technique for the treatment of a case series of congenital distichiasis carriers treated at King Khaled Eye Specialist Hospital (KKESH), Riyadh, Saudi Arabia.

\section{Methods}

A case series of three patients are presented to illustrate the technique and describe the clinical and histologic features and the postoperative outcomes.

\section{Surgical technique}

The technique is presented in Figure 1. Surgery can be performed under general (for children and uncooperative patients) or local anesthesia. Local anesthesia performed with infiltration of lidocaine $2 \%$ and epinephrine 1:200.000 (Xylocaine, Lake Forest, IL, USA) in the affected lid. A large chalazion clamp is used to secure the affected area. A 15 Parker blade is used to horizontally split the lid margin, extending to the entire region containing the distichiatic follicles, anteriorly to the orifices of the abnormal lashes. Subsequently, $3 \mathrm{~mm}$ to $4 \mathrm{~mm}$ superior dissection is performed to separate the posterior lamella. Once the affected area is isolated, a tarsectomy in the posterior lamella containing the distichiatic lashes is horizontally excised. Then, the excised area is measured and a composite tarsoconjunctival graft that is $1 \mathrm{~mm}$ larger than the exposed area created in the lid margin is obtained from the superior end of the tarsus, respecting the levator aponeurosis. The donor area does not require suturing. The graft is sutured with uninterrupted 7-0 Vicryl polyglactin (Vicryl; Ethicon, Somerville, NJ, USA) in the area exposed by the marginal tarsectomy, replacing the lid margin where the distichiatic lashes were excised. Positioning of the lashes and lid margin can be performed at the end of the procedure. An ophthalmic ointment containing a combination antibiotic and steroid is applied along the lid margin and a firm pressure patch is placed over the eye for 24 hours. 


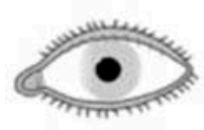

$1 \mathrm{~A}$

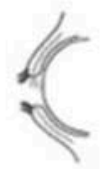

$1 \mathrm{~B}$

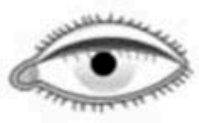

$2 \mathrm{~A}$

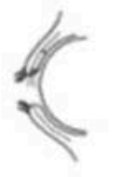

$2 B$

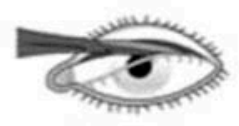

$3 \mathrm{~A}$

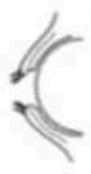

3B

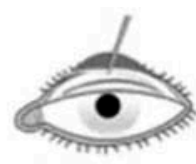

$4 \mathrm{~A}$
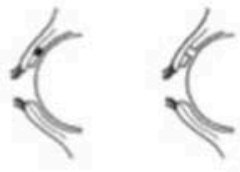

$4 B$

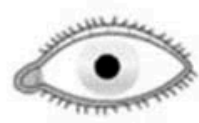

$5 A$

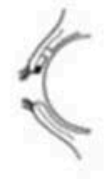

$5 B$

Figure 1. Schematic drawing of the steps for the split lamellae, marginal tarsectomy with tarsoconjuntival graft technique. A. (upper line): coronal view; B. (lower line): sagittal view. 1A-1B. distichiatic lashes, 2A-2B. split lamellae - a superior dissection is performed to separate the distichiatic row of lashes from the anterior lamellae. The lid margin is split just anterior to the orifices of the abnormal distichiatic follicles. 3A-3B. the distichiatic row of lashes and the posterior lamellae are removed (horizontal marginal tarsectomy). $4 \mathrm{~A}, 4 \mathrm{~B}$. The upper lid is everted and the tarsoconjunctival graft is marked and excised. $5 \mathrm{~A}-5 \mathrm{~B}$. The tarsoconjunctival graft is placed in the marginal tarsectomy.

\section{Case reports}

Case 1: A 4 year-old female presented with chief complaints of a rubbing sensation from the lashes and redness on her left upper lid since birth. Ophthalmic examination indicated a densely grouped tuft of lashes on the outer side of her left upper lid margin, $2 \mathrm{~mm}$ below the lash line coinciding with the Meibomian gland orifices. Distichiatic cilia had been present since birth, and the lashes were thinner, lightly pigmented and were periodically manually epilated by the parents. The lid margin was slightly pigmented. Eversion of the upper lid indicated the tarsal and bulbar conjunctiva were unaffected and there were no signs of inflammation. There was no family history of a similar presentation or any other systemic disorder. The patient underwent excisional removal of the sectorial distichiatic lashes from the upper left lid and a composite tarsoconjunctival graft was placed from same upper lid. At 9 months postoperatively, the patient was stable with complete healing of the left upper lid margin and satisfactory lid position and contour.

Case 2: A 7 year-old male with a known diagnosis of congenital distichiasis presented with complaints of occasional episodes of photophobia and discharge. At presentation, he had keratinization of the lid margin, and abnormal thin, shorter and discolored lashes emerging from the Meibomian glands ostia in all eyelids. The abnormal lashes from the upper lids were causing corneal abrasions and there was positive fluorescein staining bilaterally. No lymphedema or other systemic abnormalities were detected. He had other affected first-degree relatives (father and 2 brothers) with the same disease. Genetic studies for FOXC2 were negative.
The abnormal hairs were removed manually several times and the patient had undergone multiple sessions of electrolysis cauterization under general anesthesia. We elected to perform a tarsoconjunctival graft in both upper lids. The graft was obtained from the same side of each upper lid. At 9 months postoperatively, both upper lids margins were completely healed with good surgical outcome and no lashes were rubbing the cornea.

Case 3: A 12 year-old male with congenital distichiasis presented with complaints of discharge, photophobia and foreign body sensation. He was the older sibling of case 2 above. The patient had abnormal thin, short and less pigmented lashes in his left upper lid since birth. The left cornea had superficial scars superiorly and recent epithelial defects with positive fluorescein staining, dry periocular skin with very distorted margin and keratinization (Figure 2A). He had a history of previous multiple sessions of manual epilation of these lashes, repeated electrolysis and two entropion surgical repair procedures (lid margin rotation and split lamellae with anterior lamellar repositioning). Genetic testing for FOXC2 was negative. There was no other ocular or systemic abnormalities. We performed a composite tarsoconjunctival graft on the left upper lid in the affected area. The graft was obtained from the same upper lid (Figure 2B) At 9 months postoperatively, the lid margin was completely healed lid with a satisfactory position, contour and no abnormal lashes.

\section{Discussion}

In this case series presents a novel surgical procedure for treating congenital distichiasis. Two patients were negative for genetic testing for lymphedema-distichiasis 

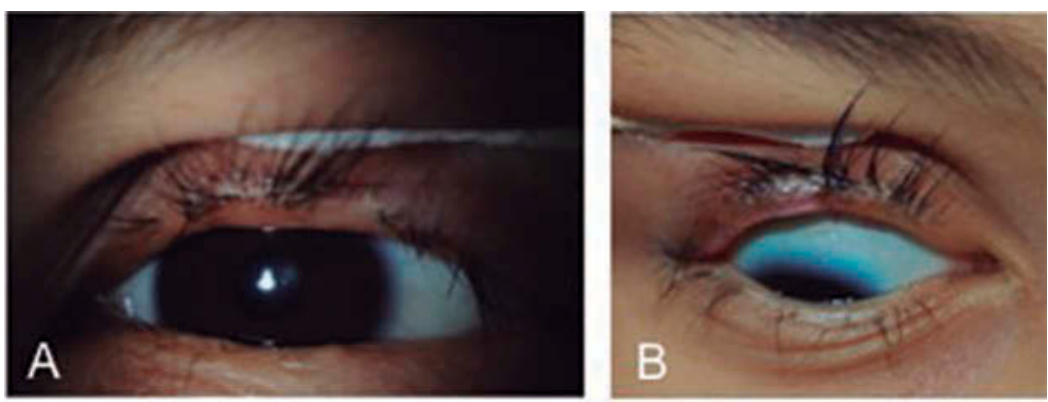

Figure 2. A.-B Case 3. A, Preoperative clinical picture, Thin and deformed upper lid, no lashes are seen due to recent epilation by the patient. B Appearance at 3 months postoperatively showing almost normal thickness of upper lid margin and no abnormal lashes, the upper lid with graft and stitches in the lateral region.

syndrome. Cases 2 and 3 were siblings, with an affected father and another brother. Notably, the manifestation of the disease was different in both siblings, with case 2 presenting with all lids affected and case 3 with only one affected lid.

All the patients had abnormal lashes since birth, which were characteristically shorter, thinner and less pigmented than normal lashes. This observation is consistent with features of congenital distichiasis. ${ }^{12}$ Due to the characteristics of the lashes, severe symptoms are rare. Symptoms can progress after unsuccessful procedures, generally after several manual epilation sessions. After these repeat epilation sessions, the lashes can regrow thicker and abrade cornea or conjunctiva increasing the risk of keratitis and corneal ulcers.

The decision to recommend surgery in the three patients presented in this study was based on the symptoms, significant corneal staining or ulceration, extent of lid margin involvement and the history of unsuccessful treatment with manual epilation (case 1), several epilation/electrolysis (cases 2 and 3) sessions, or unsuccessful previous surgery (case 3 ).

Manual epilation with or without a microscope will result in regrowth in all the treated lashes. ${ }^{3,4}$

Electrolysis ${ }^{4}$ or cryotherapy of the lid margin or only over the posterior lamella after a split lamella can result in skin depigmentation, fibrosis and distortion of the tarsal plate, causing entropion and trichiasis. ${ }^{1,5}$ Over half of these patients may require retreatment. ${ }^{13}$ In the current study, both patients who were treated with electrolysis presented with distorted lid margins and a small, deformed tarsus. Additionally, the destruction of the structures from the tarsal plate was confirmed with histopathology. However, the aberrant lashes continued to regrow in both patients.

The pathogenesis of distichiasis remains controversial. In congenital distichiasis, a developmental anomaly occurs in which a complete pilosebaceous unit(s) (with hair and glandular structures) is present in the posterior lamellae. This likely represents a failure of the primary epithelial cells to selectively differentiate into a sebaceous gland only. Instead, it develops into a complete pilosebaceous unit, in which case it would not represent a true metaplasia as has been previously suggested. However, we cannot completely eliminate the possibility of an in-utero injury leading to metaplasia in congenital distichiasis, but this seems unlikely. ${ }^{5}$

We suggest a surgical procedure to treat distichiasis involving split lamellae, marginal tarsectomy and a composite graft. Other surgical procedures have been proposed to treat distichiasis including individual lash root excision, incision of distichiatic lash root, or distichiasis eyelash removal through a tarsoconjunctival trapdoor. ${ }^{1,8}$ However, all these procedures are time consuming and the outcomes are variable. ${ }^{1,8}$

The split lamellae technique along the grey line has been previously described, with anterior lamellae repositioning, sliding or resecting the lamella with the lashes or using nasal chondro-mucosa or buccal mucous membrane grafts into the split lid margin, in order to keep the lashes distant from the globe. ${ }^{14}$

Our technique used a composite tarsoconjunctival graft, removed from the same lid, with the donor area near the distichiatic region. The other grafts suggested in the literature require two surgical sites and can cause more morbidity. Another advantage the reconstruction of the lid margin is performed with tissue that is very similar to the resected tissue. In our technique, the graft is composed of tarsus and conjunctiva which presents two major advantages. Firstly, the tarsus provides structural support to the lid margin and the conjunctiva. Secondly, the conjunctiva remains facing the globe and replaces the excised conjunctiva. In our cases, the gap between the donor area and the superior margin of the tarsus re-epithelialized without apparent contraction despite the absence of a suture. The re-epithelialization avoided elevation of the lid. A similar technique was described 
to treat upper-lid cicatricial entropion involving tarsal grafting and marginal tarsotomy and was considered a simple and effective procedure. ${ }^{15}$

A limitation of this technique is that it cannot be used for abnormalities in the upper tarsus which is the tarsoconjunctival donor area.

In the current series postoperative follow-up at 9 month indicated stable results. There were no complications such as entropion or lid margin distortion in our series. Most likely, the tarsal graft acts as a mechanical barrier to inward rotation of the lid margin and lashline. $^{15}$

We believe this technique has several advantages and could be used in cases of acquired distichiasis, with the possibility of replacing the fibrotic tarsal plate due to previous treatments with normal, similar tissue. It is very important to increase the number of cases using this interesting new technique to expand knowledge about this.

In conclusion, the split lamellae with a composite tarsoconjunctival graft offer the ideal donor material to replace tissues in the affected lid. This technique allows access the distichiatic lashes with a donor site in the same region. The major advantages of this technique over previously techniques include, preservation of normal lashes, no risk of cicatricial entropion and replacement of the damaged marginal tarsus.

\section{Disclosure statement}

Disclosure of potential conflicts of interest: The authors declare that they have no conflict of interest.

\section{Compliance with ethical standards}

Ethical approval: This article does not contain any studies with animals performed by any of the authors.

Informed consent: For this type of study formal consent is not required.

\section{References}

1. O’Donnell BA, Collin JR. Distichiasis: Management with cryotherapy to the posterior lamella. $\mathrm{Br} J$ Ophthalmol. 1993;77(5):289-292.

2. Fox SA. Distichiasis. Am J Ophthalmol. 1962;53:14-18.

3. Wolfley D. Excision of individual follicles for the management of congenital distichiasis and localized trichiasis. J Pediatr Ophthalmol Strabismus. 1987;24 (1):22-26.

4. Scheie HG, Albert DM. Distichiasis and trichiasis: Origin and management. Am J Ophthalmol. 1966;61 (4):718-720.

5. Anderson RL, Harvey JT. Lid splitting and posterior lamella cryosurgery for congenital and acquired distichiasis. Arch Ophthalmol. 1981;99(4):631-634.

6. Bedford PG. Distichiasis and its treatment by the method of partial tarsal plate excision. J Small Anim Pract. 1973;14(1):1-5.

7. Delaney MR, Rogers PA. A simplified cryotherapy technique for trichiasis and distichiasis. Aust $J$ Ophthalmol. 1984;12(2):163-166.

8. Dortzbach RK, Butera RT. Excision of distichiasis eyelashes through a tarsoconjunctival trapdoor. Arch Ophthalmol. 1978;96(1):111-112.

9. Fein W. Surgical repair for distichiasis, trichiasis, and entropion. Arch Ophthalmol. 1976;94(5):809-810.

10. Frueh BR. Treatment of distichiasis with cryotherapy. Ophthalmic Surg. 1981;12(2):100-103.

11. Handzel DM, Feretos C, Aral H. Treatment of trichiasis and distichiasis with a biopsy punch - an effective and practical method for eyelash follicle excision. Klin Monbl Augenheilkd. 2013;230(1):43-45.

12. Byrnes GA, Wilson ME. Congenital distichiasis. Arch Ophthalmol. 1991;109(12):1752-1753.

13. Sarkar S, Bagchi K, Mukherjee G. Congenital distichiasis treated by a time-based cryosurgical technique-a case report. I Indian Med Assoc. 2012;110 (10):747-748.

14. White JH. Correction of distichiasis by tarsal resection and mucous membrane grafting. Am J Ophthalmol. 1975;80(3 Pt 2):507-508.

15. Baylis HI, Hamako C. Tarsal grafting for correction of cicatricial entropion. Ophthalmic Surg. 1979;10 (7):42-48. 